\title{
DESIGN OF SELECTION EXPERIMENTS FOR COMPARING ALTERNATIVE TESTING REGIMES
}

\author{
WILLIAM G. HILL \\ Institute of Animal Genetics, Edinburgh EH9 $3 \mathrm{NN}$
}

Received 26.v.78

\begin{abstract}
SUMMARY
Selection response achieved in lines selected in different environments, for example feeding regimes, can be evaluated in two ways. Responses can be compared in a single environment, perhaps one of the test regimes or a commercial feeding regime. Alternatively, a test for interaction can be conducted in which both selected lines are tested on both selection regimes. The variances of direct and correlated responses are compared. It is shown that if there is a high genetic correlation (small interaction) in performance on the selection regimes, a high correlation due to drift is induced between the performances of a line on two environments. This enables a much more precise test to be conducted of interaction than of differences in response on a single environment.
\end{abstract}

\section{InTRODUGTION}

Selection experiments run for a few generations can give efficient estimates of genetic parameters both for the traits selected and other traits correlated with them. Although the errors in measuring genotypic from phenotypic means become trivial as they become spread over several generations of selection, the random genetic drift in the selected lines accumulates (Hill, 1971). One specific use of selection experiments is to compare the efficiency of different methods of selection or selection in different environments; for example two test feeding regimes might be compared for improving performance on a commercial regime. A related use of selection experiments is to specifically test for genotype $\times$ environment interaction, say among these test feeding regimes. Some of these problems have been discussed before (Hill, 1971), but an important difference between the error structures of these experiments was overlooked. As will be shown in this note, the test for interaction in which two selection regimes are evaluated in a cross-over experiment can be conducted with much more precision than a comparison of the two selection regimes on a single terminal regime. The formulation will initially be made more general, but the discussion is focused on this problem.

\section{THEORY}

For trait $j$ (or performance of a given trait on environment $j$ ), let $h_{j}^{2}$ be its heritability, $\sigma_{j}$ its standard deviation and $c_{j}^{2}$ the proportion of variance due to common environment of full-sibs, i.e. Cov (full sibs) $=\left(c_{j}^{2}+\frac{1}{2} h_{j}^{2}\right) \sigma_{j}^{2}$. For traits (environments) $j$ and $k$ let $r_{G_{j k}}$ be the (additive) genetic correlation, $r_{C_{j k}}$ the correlation of full sibs due to common environment and $r_{P_{j k}}$ be the phenotypic correlation, defined only if records on both traits can be taken on individuals. 
Selection is assumed to be practised on only one trait in each line for $t$ generations. In the line selected on trait $j$, let $i_{j}$ be the standardised selection differential and $\mathcal{N}_{j}$ be the effective population size, both assumed constant each generation. When a line is tested for both traits $j$ and $k$, let $T_{j k}$ be the number of animals tested for both $\left(T_{j k}=0\right.$ for two environments) and $D_{j k}$ be the number of full-sib families with progeny tested for both, with equal numbers per family. In the line selected on trait $j$, the observed response on trait $k$ is $R_{j k}$. Response is, of course, a function of $t$ but, for brevity, a subscript $t$ is ignored.

A number of simplifying assumptions will be made so as to produce tractable formulae, and the shorter the duration of the experiment, the more likely are these to hold. There is assumed to be no inbreeding depression and no changes in within-line parameters, such as variances, heritabilities and correlations, occur as a result of selection or drift. The variance among conceptual replicated selected lines is taken to equal that among unselected lines of equivalent size, and for computing this variance gene action is assumed to be additive. In earlier papers (Hill, 1971, 1974) more complete formulae have been given for variance among lines, but in view of many other assumptions made in their derivation, among these ignoring the effect of selection on variance noted by Bulmer (1971), simpler formulae will be used here. A fuller discussion of formulae and their simplifications has been given previously (Hill, 1977).

The expected response (i.e. over a series of replicate populations) in trait $k$ from selecting for trait $j$ is

$$
E\left(R_{j k}\right)=t i_{j} h_{j} h_{k} r_{G j k} \sigma_{k}
$$

(Falconer, 1960). The variance in response comes from two distinct parts: (i) genetic drift in the selected line, which depends on the effective number of parents each generation, and (ii) sampling of a limited number of individuals for testing, which depends on variation within families about their genetic mean and on common environment effects. Thus

$$
V\left(R_{j k}\right)=\left[t h_{k}^{2} / N_{j}+c_{k}^{2} / D_{k k}+\left(1-c_{k}^{2}-\frac{1}{2} h_{k}^{2}\right) / T_{k k}\right] \sigma_{k}^{2} .
$$

If performance on the two traits is measured on the same animals at the end of the experiment

$$
\begin{aligned}
\operatorname{Cov}\left(R_{j k}, R_{j l}\right)=\left[t h_{k} h_{l} r_{G k l} / N_{j}+c_{k} c_{l} r_{C k l} / D_{k l}\right. \\
\\
\left.+\left(r_{P}-c_{k} c_{l} r_{C k l}-\frac{1}{2} h_{k} h_{l} r_{G k l}\right) / T_{k l}\right] \sigma_{k} \sigma_{l} .
\end{aligned}
$$

If different animals are sampled, but are members of the same full-sib families, the terms involving $T_{k l}$ drop out of (3); and if they are from different full-sib families, the term involving $D_{k l}$ also drops out. Note that (1)-(3) include cases of direct selection (response $=R_{j j}$ ) and covariances of direct and correlated effects, e.g. $\operatorname{Cov}\left(R_{j j}, R_{j k}\right)$, simply by setting the appropriate correlations to unity. Assuming lines selected for different traits are maintained genetically separate, there is no covariance of their responses, i.e.

$$
\operatorname{Cov}\left(R_{j l}, R_{k m}\right)=0, j \neq k,
$$

eq. (4) holding even if $l=m$

Subsequently, for simplicity, it will be assumed that $\mathcal{N}_{j}=\mathcal{N}, D_{j k}=D$ and $T_{j k}=T$ for all $j$ and $k$. 


\section{Alternative testing Regimes}

Let us contrast the precision of experiments comparing responses in one environment with an interaction test where they are compared in two. With selection practised on environments 1 and 2 and the terminal response measured in 3, the ratio of mean to standard error is, from eq. (1), (2) and (4), since the responses in the lines are independent,

$$
\frac{E\left(R_{13}-R_{23}\right)}{S E\left(R_{13}-R_{23}\right)}=\frac{t\left(i_{1} h_{1} r_{G 13}-i_{2} h_{2} r_{G 23}\right) h_{3}}{\left\{2\left[t h_{3}^{2} / N+c_{3}^{2} / D+\left(1-c_{3}^{2}-\frac{1}{2} h_{3}^{2}\right) / T\right]\right\}^{\frac{1}{2}}} .
$$

A simple case of particular interest, which is also useful for illustration, is where the final test is on one of the selection regimes, say 1 , and where there are assumed to be no differences in heritability or selection intensity, i.e. $h_{1}^{2}=h_{2}^{2}, i_{1}=i_{2}$. For further simplification, assume $c_{1}^{2}=0$. Then, after dropping unnecessary subscripts, (5) reduces to

$$
x=\frac{E\left(R_{11}-R_{21}\right)}{S E\left(R_{11}-R_{21}\right)}=\frac{t i h^{2}\left(1-r_{G}\right)}{\left\{2\left[t h^{2} / N+\left(1-\frac{1}{2} h^{2}\right) / T\right]\right\}^{\frac{1}{2}}} .
$$

In general, the lack of interaction between testing regimes has to be assessed from the genetic correlation estimate. Falconer (1960) gives the following estimator for a double selection experiment, in which a line is selected for trait (environment) 1 and another for trait 2, and response on the alternative trait assessed on each line,

$$
\hat{r}_{G}=\hat{r}_{G 12}=\left[\left(R_{12} R_{21}\right) /\left(R_{11} R_{22}\right)\right]^{\frac{1}{2}} .
$$

Because $\hat{r}_{G}$ from (7) is a ratio, approximate methods are necessary for finding its standard error (Hill, 1971). If, however, in the special case where selection intensities and heritabilities can be assumed equal in both environments, the usual interaction contrast from the analysis of variance, $R_{11}-R_{12}-R_{21}+R_{22}$, can be used. Making other assumptions as for (6), and noting that no animals are tested on both environments, from (2) and (3)

$$
V\left(R_{11}-R_{12}\right)=2\left[\left(1-r_{G}\right) t h^{2} / N+\left(1-\frac{1}{2} h^{2}\right) / T\right] \sigma^{2} .
$$

As seen from (8), if there is a high genetic correlation over environments, this reduces the variance in the difference of response on a line over the two environments. This point is further illustrated by considering the usual contrasts from the $2 \times 2$ cross classification. Letting $\sigma_{d}^{2}=h^{2} \sigma^{2} / \mathcal{N}$ and $\sigma_{e}^{2}=\left(1-\frac{1}{2} h^{2}\right) \sigma^{2} / T$, then the mean response/line is:

$$
V\left(R_{11}+R_{12}-R_{21}-R_{22}\right)=4\left[t\left(1+r_{G}\right) \sigma_{d}^{2}+\sigma_{e}^{2}\right],
$$

the mean response/test environment is:

$$
V\left(R_{11}+R_{21}-R_{12}-R_{22}\right)=4\left[t\left(1-r_{G}\right) \sigma_{d}^{2}+\sigma_{e}^{2}\right],
$$

and the interaction is: $V\left(R_{11}-R_{12}-R_{21}+R_{22}\right)=4\left[t\left(1-r_{G}\right) \sigma_{d}^{2}+\sigma_{e}^{2}\right]$. The sensitivity of the interaction test is therefore

$$
y=\frac{E\left(R_{11}-R_{12}-R_{21}+R_{22}\right)}{S E\left(R_{11}-R_{12}-R_{21}-R_{22}\right)}=\frac{\operatorname{tih}^{2}\left(1-r_{G}\right)}{\left[\left(1-r_{G}\right) t h^{2} / N+\left(1-\frac{1}{2} h^{2}\right) / T\right]^{\frac{1}{2}}},
$$


which differs from (6) in the absence of a term of $\sqrt{2}$ since four rather than two lines are involved, in addition to the reduction in variance caused by the drift correlation.

To compare the direct (6) and interaction (9) tests, assume the selected lines are maintained at the same size, with $s$ sires and $d$ dams/sire so, ignoring any selection influence on effective population size, $1 / \mathcal{N}=1 /(4 s)+1 /(4 s d)$, and that the final comparison is made with $n_{x}$ or $n_{y}$, respectively, progeny per litter, so $\mathrm{T}=s d n_{x}$ or $s d n_{y}$. Then, from (6) and (9)

$$
\frac{y}{x}=\left\{\frac{2\left[\operatorname{th}^{2}(d+1) / 4+\left(1-\frac{1}{2} h^{2}\right) / n_{x}\right]}{\left[t h^{2}\left(1-r_{G}\right)(d+1) / 4+\left(1-\frac{1}{2} h^{2}\right) / n_{y}\right]}\right\}^{\frac{1}{2}}
$$

In table 1 , values of $x, y$ and $y / x$ are given for some examples relevant to pigs. Values of $i$ are computed assuming three pigs per litter of each sex

TABLE 1

Values of $\mathrm{x}=\mathrm{E}\left(\mathrm{R}_{11}-\mathrm{R}_{21}\right) / \mathrm{SE}\left(\mathrm{R}_{11}-\mathrm{R}_{21}\right), \mathrm{y}=\mathrm{E}\left(\mathrm{R}_{11}-\mathrm{R}_{12}-\mathrm{R}_{21}+\mathrm{R}_{22}\right) / \mathrm{SE}\left(\mathrm{R}_{11}-\mathrm{R}_{12}-\mathrm{R}_{21}+\right.$ $\mathrm{R}_{22}$ ) and $\mathrm{y} / \mathrm{x}$ for a range of examples. Proportions selected are $1 / 3$ in females and $1 / 3 \mathrm{~d}$ in males.

$\begin{array}{rrrrrr}n_{x} & n_{y} & & \overbrace{x \times \sqrt{s}}^{-} & 0.5 \times \sqrt{s} & y / x \\ 8 & 4 & 0.790 & 1.401 & 1.77 \\ 8 & 4 & 0.650 & 1.127 & 1.73 \\ & & & & \\ 8 & 4 & 0.876 & 1.638 & 1.87 \\ 8 & 4 & 0.909 & 1.638 & 1.80 \\ 8 & 8 & 0.909 & 1.751 & 1.93 \\ 8 & 16 & 0.909 & 1.817 & 2.00 \\ 8 & 4 & 1.144 & 2.140 & 1.87 \\ 8 & 4 & 0.947 & 1.746 & 1.84\end{array}$

are available for selection (after culling for legs etc.), so the proportion selected is $1 / 3$ for females and $1 / 3 \mathrm{~d}$ for males. Finite population corrections to $i$ have been ignored, so both $x$ and $y$ are proportional to $\sqrt{s}$.

The relative efficiency, $y / x$, is seen from table 1 to increase markedly as the genetic correlation becomes high. It also rises as the number of generations or the number tested at the end are increased, since in each case genetic drift comprises a larger part of the total variance. The interaction test involves twice as many tested pigs as the direct comparison if the number tested on each line $\times$ environment combination is the same $\left(n_{x}=n_{y}\right)$. Thus in the interaction test a higher proportion of total facilities should be devoted to the final comparison, but this could simply involve use of second litters.

The values in table 1 can also be used to compute sizes of experiment, using the procedure of Hill (1971). For example, if it is desired to test whether the correlation is less than 0.8 using an interaction test, then with reasonable power and significance level, the value of $y$ should exceed about 2.5. For example, for $t=4, d=2, n_{y}=8$ and $r_{G}=0.9$, from table 1 $=0.625 \sqrt{s}$. Hence $s$ should be at least $(2 \cdot 5 / 0 \cdot 625)^{2}=16$. 


\section{Discussion}

The alternative types of experiment described here answer two different questions and their efficiencies can not strictly be compared as if they were alternative ways of answering the same question. The analysis shows, however, that if the problem can be posed as just a test for interaction, a much smaller experiment can be run than if the efficacy of two alternative selection regimes have to be compared, say on a commercial diet.

A very simplified structure of the selection experiments has been considered; there are numerous ramifications but these would probably have obscured the main point. For example, testing on the alternative regime could be carried out on one or more generations prior to the termination of the experiment. This would add somewhat to efficiency. Divergent selection could be practised, in which one line is selected high and one low on each regime. This is feasible if there is assumed to be no asymmetry. Then the expected responses (high-low) are doubled relative to those shown, and if the same total facilities are split into twice as many lines, variances of response/line are doubled and thus of total response quadrupled.

Hence $x, y$ and $y / x$ are not affected. If a control population is maintained facilities have to be diverted from the selected lines and the efficiencies $x$ and $y$ are reduced (see Hill (1972) for further discussion). A control population or divergent line is necessary if the mean rate of response has to be estimated so that the interaction or difference in response can be put in relative terms. The control or divergent line could be omitted, however, if rates of response on one test regime were already well known.

The interaction comparison $R_{11}-R_{12}-R_{21}+R_{22}$ can only be used if variances and heritabilities are the same. If, as is likely, this is questioned in any experiment then the correlation estimate (7) has to be used. Its sampling error, while more complicated and approximate, is similar to that given here for the interaction test (Hill, 1971). Terms in $1-r_{G}$ appear in the variance, just as they do for genetic correlations estimated from the covariance of half-sibs (Robertson, 1959). As an alternative to the interaction test, phenotypic standard deviations and selection intensities can be scaled out in a contrast such as $R_{11} / i_{1} \sigma_{1}-R_{12} / i_{1} \sigma_{2}-R_{21} / i_{2} \sigma_{1}+R_{22} / i_{2} \sigma_{2}$, but the heritability can not so easily be eliminated. The important point is, however, that the variance of the interaction test or genetic correlation estimate drops as the correlation rises, whereas the direct test of two selection regimes on one environment is not so improved.

Acknowledgements.-I am grateful to Dr Charles Smith for many helpful comments.

\section{REFERENGES}

BULmer, M. G. 1971. The effect of selection on genetic variability. American Naturalist, $105,201-211$.

FALCONER, D. S. 1960. Introduction to Quantitative Genetics. Oliver and Boyd, Edinburgh. HILL, W. G. 1971. Design and efficiency of selection experiments for estimating genetic parameters. Biometrics, 27, 293-311.

HILL, W. G. 1972. Estimation of realised heritabilities from selection experiments. II Selection in one direction. Biometrics, 28, 767-780.

HILL, W. G. 1974. Variability of response to selection in genetic experiments. Biometrics, $30,363-366$. 
HILL, W. G. 1976. Variation in response to selection. Proc. Int. Conf. Quantitative Genetics, ed. E. Pollak, O. Kempthorne and T. B. Bailey, pp. 343-365. Iowa State Univ. Press, Ames.

ROBERTSON, A. 1959. The sampling variance of the genetic correlation coefficient. Biometrics, 15, 469-485. 